\title{
A Maximal Mass Confinement Principle for Rigid and Locally Rigid Image Registration
}

\author{
Julian Mattes, Johannes Gall, and Alfredo Lopez
}

Biomedical Data Analysis Group

KBT-Area, Software Competence Center Hagenberg, 4232 Hagenberg, Austria Vidiatech Hagenberg, Softwarepark 35, 4232 Hagenberg, Austria

Julian.Mattes@scch.at

\begin{abstract}
In this paper we propose (1) to set the problem of image registration as a contour/region-template-to-image matching problem using so-called confiners - also called blobs or components - as template regions, (2) to select the confiners of one of the images by passing through the hierarchical structure which they define and registering them successively rigidly form coarse-to-fine to the other image, the target image, and (3) we propose a maximum mass confinement (MMC) principle for contour-to-image registration. This principle allows us to derive a similarity measure assessing how well the confiner fits into the target image simply by calculating the gray value mass confined by its contour. By optimizing this measure for rigid transformations we obtain our MMC algorithm registering a contour locally rigid to the target image. We illustrate that by proceeding based on (1-3) problems can be avoided which were related to previous registration algorithms based on confiners. We compare our MMC algorithm with another template matching algorithm based on normalized mutual information. Equally, we compare our hierarchical image registration strategy with B-Spline based non-rigid registration using normalized mutual information. We performed our evaluation on real and simulated images in terms of robustness, accuracy and computation speed. We show that both, MMC template matching on its own and hierarchical image registration using MMC, in most cases outperform the respective alternative method.
\end{abstract}

\section{Introduction}

Image registration is defined as the process of determining the spatial transformation relating physically or anatomically corresponding points in two images or to bring them together as close as possible for a given class of transformations. It has a large number of applications, for instance, to bring different images of the same object/person into the same reference system, to compare anatomically corresponding objects of different subjects, or to determine the motion appearing from one image to the next one within a time series. Various methods for rigid and non-rigid image registration have been proposed during the past 20 years. Many of them are using an iterative process to transform one image onto 
the other and define a similarity measure assessing at each iteration how well the fixed and the transformed image are resembling.

These methods differ among others with respect to the image features which are used to define the similarity measures. So-called iconic measures compare gray intensity values directly. An example is the mutual information measure which has been widely used in different fields of applications [1. On the other hand, there are methods comparing previously extracted features which can be defined at different levels, for instance, by incorporating gradient information or by using unconnected edges or, at a high level, segmented objects.

An example for features at an intermediary level are called blobs (also called components, or confiners), the connected image regions obtained after thresholding, and their contours or surfaces, the iso-lines or -surfaces. These regions can be efficiently extracted and different approaches have been proposed to identify corresponding (physically or anatomically) blobs in both images [2]3] and to base the registration process on them. However, small differences in both images (e.g., because of noise) can lead to considerable changes of the blobs of the respective images, even though parts of them may still correspond. Therefore, often only very few (or no) well corresponding blobs can be detected.

In this paper we propose to avoid the problems associated with the identification of corresponding blobs by setting the registration task as a hierarchical contour-to-image template matching problem and by defining a new similarity metric for contour- (or region-) to-image matching. Our similarity measure is based on a maximal mass confinement principle which we introduce in the next section. We show that this measure and strategy lead to a simple but efficient method not only for contour-to-image matching but also for rigid and locally rigid image registration usually able to cope with large local movements. Thereto, we evaluate our approach on several real and synthetic image pairs and compare it with a registration strategy using normalized mutual information (Section 3). We found the idea of volume image registration by template matching also in 4], however, well known methods (e.g., based on mutual information) have been used to register template regions - not confiners but specific cubic and spherical subvolumes have been used - of one image to the other image.

\section{Maximal Mass Confinement for Template-to-Image Registration}

We introduce in this section a new principle for region- (or contour-/surface-) to-image registration, from which we derive an image registration algorithm. Our principle follows from a property of the so-called level sections (or cross sections) of an image and of its connected components. Both we introduce next.

Given the intensity function $f_{I}: \mathbb{R}^{d} \rightarrow \mathbb{R}_{\geq 0}, d=2$ or 3 , of an image $I$ we define a confiner (or blob) formally as a maximal connected subset (i.e., a connected component) $\mathcal{C}$ of the level section $\mathcal{L}_{l}=\mathcal{L}\left(f_{I}\right)_{l}=\left\{x \in \mathbb{R}^{d} \mid f_{I}(x) \geq l\right\}$ at a gray level $l$ (Fig. 1(a)). We define connectivity through the discrete $d 4$ neighborhood. We associate to each confiner its mass as an attribute inspired by 


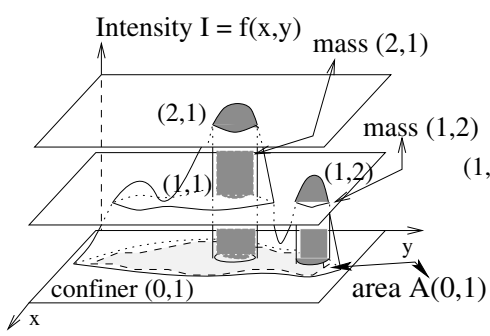

(a)

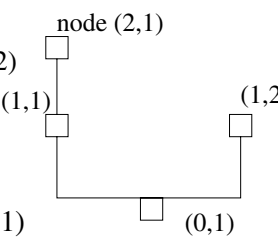

(b)

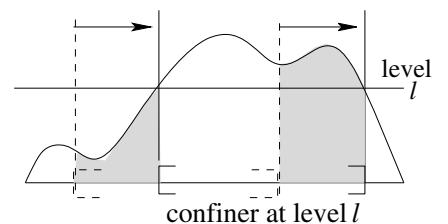

(c)

Fig. 1. Illustration of (a) the definition of the confiners, (b) the confinement tree, and (c) the confinement variational principle. By moving the region delimited by the dotted brackets in (c) onto the confiner position (solid brackets) the mass corresponding to the gray area on the left hand side is lost and that on the right hand side is gained.

measure theory; it is calculated as the sum of the gray values of the confiner's pixels. Taken for the level sections at different gray levels (including level 0) the confiners form by set inclusion a tree structure, the confinement tree (Fig. 1 (b)).

\subsection{A Mass Based Similarity Measure and the Maximal Mass Confinement Principle}

For a given integrable function $f: \mathbb{R}^{d} \rightarrow \mathbb{R}_{\geq 0}, d=2$ or 3 in our case, we define a measure $S$ which associates to each measurable subset $\mathcal{B}$ of the space $\mathbb{R}^{d}$ (i.e., the integral of $f$ over $\mathcal{B}$ exists) its mass $m_{\mathcal{B}}$ defined as the integral of $f$ over $\mathcal{B}$, hence $S(\mathcal{B})=m_{\mathcal{B}}=\int_{\mathcal{B}} f d V(d V=d x d y, f=f(x, y)$, if $d=2$ or $d V=d x d y d z, f=f(x, y, z)$, if $d=3)$. If $f=f_{I}$ is the gray intensity of an image $I$ then $\mathcal{B}$ is a set of pixels (or voxels) and $S(\mathcal{B})=\int_{\mathcal{B}} f d V$ is just the sum of each pixel's (or voxel's) gray value (see above). We denote the area (if $d=2$ or volume if $d=3$ ) of $\mathcal{B}$ with $A(\mathcal{B})$. We derived our new approach for contour-to-image template matching from the following theorem which is illustrated in Fig. 1(c).

Theorem 1. Confinement Variational Principle. Let $\mathcal{L}(f)_{l}$ be the level section of an integrable function $f: \mathbb{R}^{d} \rightarrow \mathbb{R}_{\geq 0}, d \in\{2,3\}$ and for a level $l \in \mathbb{R}$ (then $\mathcal{L}(f)_{l}$ is measurable). We set $\alpha=A\left(\overline{\mathcal{L}}(f)_{l}\right)$ (the area of $\mathcal{L}(f)_{l}$, if $d=2$ ).

Then, the mass $m_{\mathcal{L}(f)_{l}}=S\left(\mathcal{L}(f)_{l}\right)$ is maximal among all measurable subsets $\mathcal{B}_{\alpha}$ of $\mathbb{R}^{d}$ with $A\left(\mathcal{B}_{\alpha}\right)=\alpha$.

Theorem 1 holds also locally for all confiners $\mathcal{C}$ for which $f$ does not reach a local minimum or saddle point on a contour point of $\mathcal{C}$. The theorem leads directly to a template matching algorithm: For a region $\mathcal{B}$ (or for its contour) and by allowing only rigid transformations $\mathbf{r}$ the process of successively confining the maximal mass by the contour of $\mathcal{B}$ corresponds to an optimization of $S(\mathbf{r}(\mathcal{B}))$. If $\mathcal{B}=\tilde{\mathbf{r}}^{-1}(\mathcal{C})$ for a confiner $\mathcal{C}$ of the target image then the optimization of $S(\mathbf{r}(\mathcal{B}))$ allows, according to the theorem, to correctly recover $\tilde{\mathbf{r}}$ and hence the location of $\mathcal{C}$. Thus, $S$ is our similarity measure assessing how well $\mathbf{r}(\mathcal{B})$ fits 
into the target image. We call this approach maximal mass confinement (MMC) algorithm. For optimization we used in a first attempt Powell's Direction Set method. The power of the MMC principle is illustrated in Fig. 11(c). In this example the similarity is monotonously increasing until the optimal confiner location is reached, even though there are different local minima and maxima of $f$ on the way to this optimal location. Due to this robustness the MMC algorithm is able to deal with structural differences in the confiners belonging to the two images. Theorem 1 has been proposed in different settings 567] in statistics - where $f$ is a probability density function-but the use of it for template registration has not been recognized so far.

\subsection{Selection of Template Regions}

Often, the motion pattern in the images to be registered has a global component at some level of spatial resolution. Then, registering from coarse-to-fine allows a good initialization obtained from the result of coarse registration when starting the registration procedure at a finer level. We propose to register two images by selecting confiners in one image, the source image, and to register them successively by rigid template matching to the other image, the target image.

By the structure of the confinement tree, the confiners are organized in a hierarchical manner. A natural way of coarse-to-fine registration based on the confinement tree is to select the confiners hierarchically starting from the root and passing then recursively all branches originating from the first bifurcation after the root where the template matching process for a selected confiner of each branch is initialized by the transformation resulting from the matching process corresponding to the root, etc. The benefit of this strategy is illustrated in Fig. S1 (supplemental material). It leads to the following algorithm (Tsource denotes the confinement tree of the source image and Itarget denotes the target image; the rigid template matching algorithm in 3 . has been presented above):

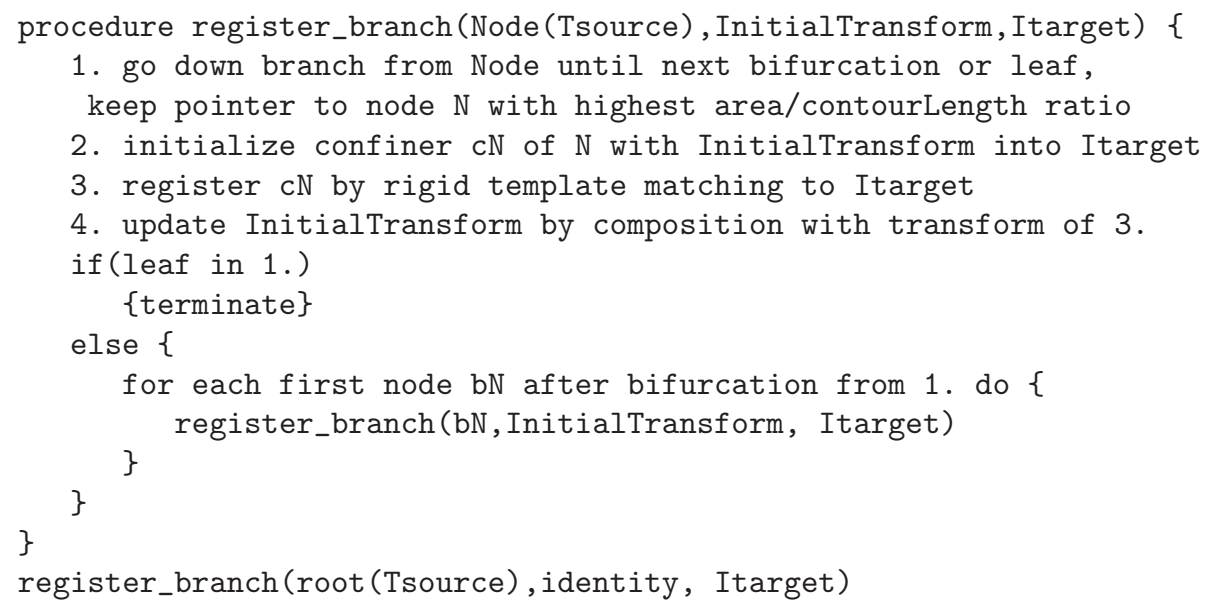




\section{Experimental Evaluation}

We evaluated first the MMC algorithm on artificially displaced template contours as detailed in the next subsection. Second, we evaluated our hierarchical image registration algorithm on real microscopic images of entire cells (Fig. 3), which show movements occurring in between two different points in time, and of cell nuclei Figs. 2, S1 (supplementary material) before and after applying a chemical procedure called Fluorescence in Situ Hybridisation (FISH). The latter we obtained from K. M. Greulich-Bode, DKFZ, Heidelberg. A further validation based on synthetic ground truth is presented in supplementary material S2.

\subsection{Robustness, Accuracy, and Speed of MMC Based Contour-to-Image Matching}

The template matching algorithm proposed in Section 2 is of interest on its own. Therefore, we evaluate it separately from our current complete confinement tree based registration method proposed in Section 2.2. We compare our MMCalgorithm with an iconic registration algorithm; in [4 mutual information based registration was proposed for iconic region-to-image template matching. We have chosen normalized mutual information (NMI) as similarity measure 8 and an image pyramid with different degrees of gaussian blurring for taken successively finer image details into account.We used the implementation available in the ITK library. We applied both algorithms to register rigidly a transformed confiner, as template region, back to the image from which the confiner has been extracted. We have taken the confiner corresponding to the upper most nucleus in the image presented in Fig. 2. Thereby, we used the confiner which is automatically selected during hierarchical confiner selection as described in Section 2.2.

In order to validate and compare the robustness and accuracy of both approaches we defined various different initializations of the template matching process obtained by transforming the selected confiner according to the following scheme: We rotated the confiner around the geometric center of its contour by $0,15,30,45$ and 60 degrees and translated it by $-200,-100,0,100$ and 200 pixels in $\mathrm{x}$ (horizontal) direction and $-75,0,75,150$ pixels in y (vertical) direction. After, we have taken all 100 possible combinations of these values in order to transform the confiner. Fig. 2 (a,c) depicts the positions of the transformed confiner's contour for selected initial transformations. For evaluating our maximal mass confinement algorithm we used only the contour of the confiner whereas for NMI template matching we kept the gray values of all pixels of the template confiner (the other pixel's gray values are set to zero).

We quantify the root mean square error $\epsilon_{r m s}$ of the pixel distances between corresponding points of the original confiner contour and the contour after registration. The error values are color coded according to the registration result for the selected initial transformations of the confiner, in Fig. 2(a) for the MMC algorithm and in Fig. 2(2) for NMI based template matching. We use the color code described in the caption of Fig. 2, green corresponds to sub-pixel accuracy (a value below 1). In the plots in Figs. 2(b,d) we indicate for each considered initial 


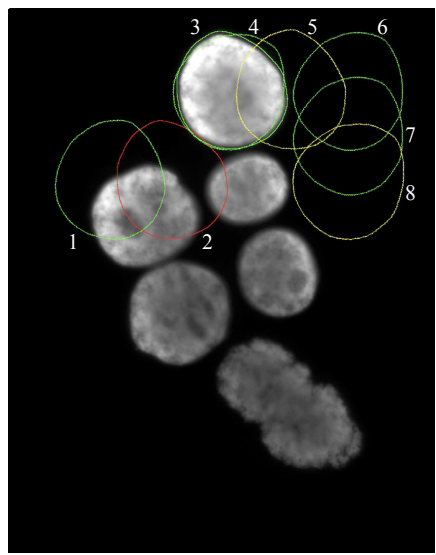

(a) Color coded and

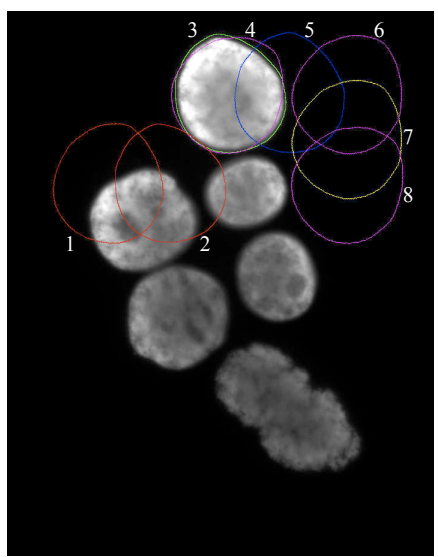

(c) Color coded and

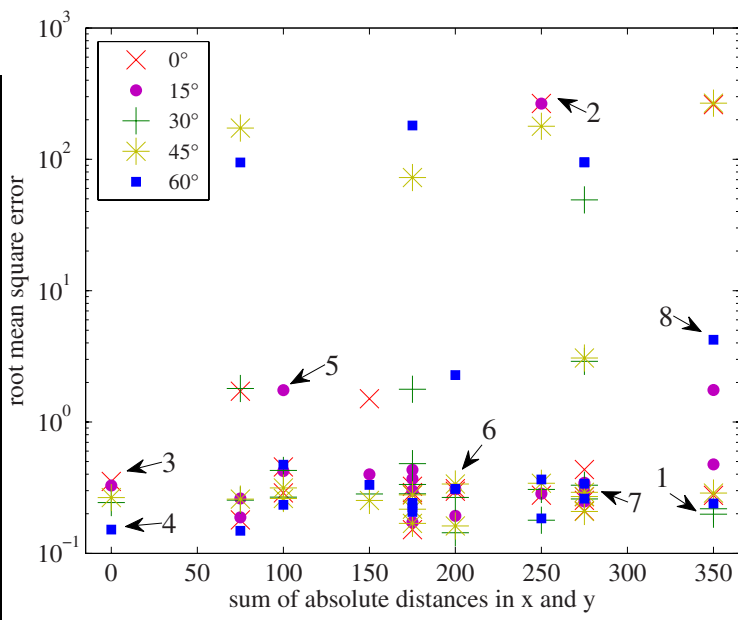

(b) plotted numerical results - MMC-algorithm

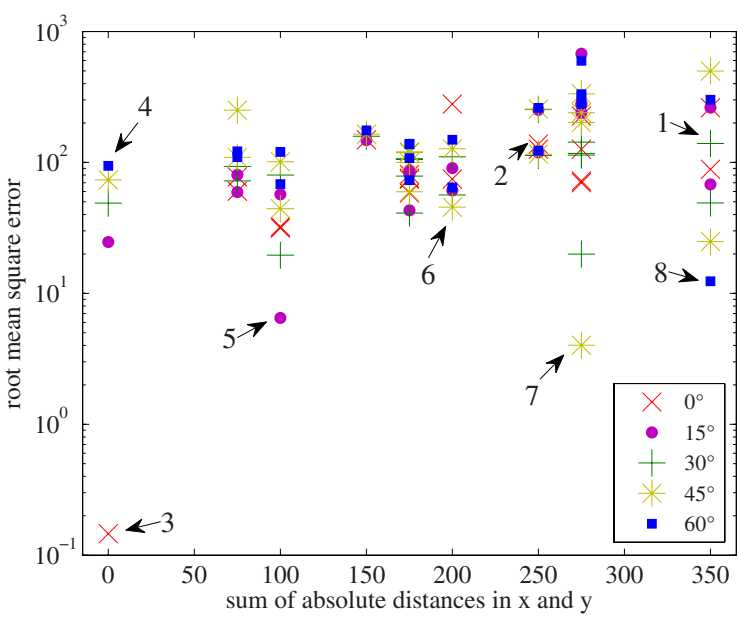

(d) plotted results - NMI-template matching

Fig. 2. Comparison of the registration results obtained with MMC and with NMI template matching for 100 different initializations of the registration process: in (a) and (c) we show the initial positions of the contour of the confiner template for selected initial transformations (see text). We coded in color the error value $\epsilon_{r m s}$ obtained after registering the template back to the (whole) initial image (see text): green $\rightarrow \epsilon_{r m s}<1$, yellow $\rightarrow 1 \leq \epsilon_{r m s}<5$, blue $\rightarrow 5 \leq \epsilon_{r m s}<10$, purple $\rightarrow 10 \leq \epsilon_{r m s}<100$, and red $\rightarrow \epsilon_{r m s} \geq 100 ;(\mathrm{b}, \mathrm{d})$ : plotted error values $\epsilon_{r m s}$ (logarithmic scale; see text) for each initial transformation where the translation is indicated by the sum of the absolute translational distances in $x$ and in $y$ and where the rotation is indicated by different symbols; a symbol with a number pointing to it corresponds to the respective initial position depicted in (a) and (c) and marked by the same number; here, the initial positions in (a) and (c) with the same numbers are identical. 


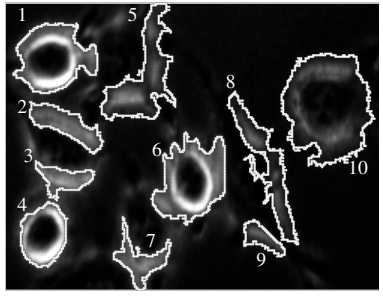

(a) source image: point in time 1

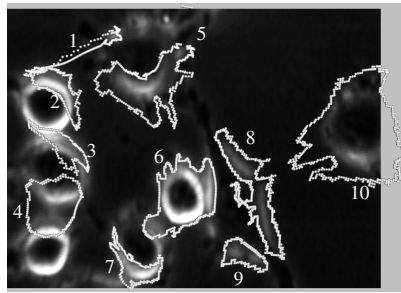

(b) target image: point in time 2 result NMI B-splines based

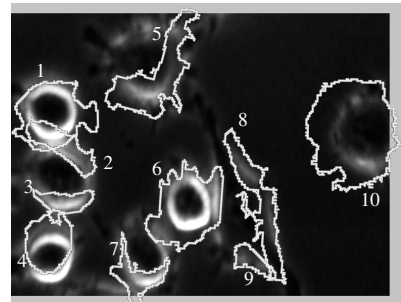

(c) target image: point in time 2 result hierarchical MMC

Fig. 3. Microscopic cell images at two different points in time: Comparison of confinement tree based registration with NMI-B-spline-registration. Contour lines are shown to visualize the result. (a): source image, the contours correspond to the hierarchically selected confiners which are closest to the leaves of the filtered tree. (b,c): target image; (b) the contours are the transformed contours of (a), transformed by registration with the NMI-B-splines based algorithm; (c) the contours are the transformed ones of (a), transformed with our hierarchical MMC algorithm. Corresponding contours have the same numbers. Note the strong deformation of the contour in the upper left corner after NMI-B-spline-registration and the misregistration of all cells on the left hand side with this approach; they have been correctly transformed by the MMC algorithm.

angle (different symbols) and for the different distances obtained by translation in $x$ and $y$ (horizontal axis) the resulting error value $\epsilon_{r m s}$ after registration. Visually, the quality of the result in the case of a green or a yellow line are difficult to distinguish.

Our results show that the maximal mass confinement method works very accurately and also robustly. In 89 of 100 cases we obtain a very good or good $\left(\epsilon_{r m s}<5\right)$ registration result among them 79 cases with sub-pixel accuracy. In contrast, using NMI template matching we obtained only for the case of a perfect initialization (no transformation) sub-pixel accuracy. 1 further case lead to a good result $\left(\epsilon_{r m s}=4.01\right)$ but in all other 98 cases a mis-registration occurred. A further benefit of the MMC approach results from the low computing costs compared to NMI template registration. Less than 1 sec of computation for complete template registration with $\mathrm{MMC}$ in case of the confiner template used in Fig. 2 compares to 1-2 min required for rigid NMI template registration.

\subsection{Comparison of Hierarchical MMC with an Iconic Approach Using a B-Spline Motion Model}

Next, we address the comparison of the hierarchical registration algorithm proposed in Section 2.2 with a typical non-rigid registration algorithm often used for motion analysis combining the use of normalized mutual information as similarity measure with bi-linear B-splines [8]. The control points of the B-splines lay on the nodes of a regular grid and are inserted using a hierarchical scheme with 10 levels. Starting with 3 points per linearly independent coordinate axis (together 9 points), 2 control points per axis are inserted in each step until 21 points per 
axis (together 441 control points) are reached. We evaluated the algorithm on the microscopic cell images depicted in Fig. 3. In cases of strong local motion of the cells, the NMI-B-splines based approach failed and often strongly deformed cells whereas the MMC based algorithm still worked robustly. Moreover, the MMC based algorithm leads to a striking gain in computation time: We need around 3 min with the approach using NMI (with image pyramid, ITK application) and B-Splines compared to around $7 \mathrm{sec}$ with the MMC based algorithm, which has still a big potential to be optimized with respect to its computation time. A more thorough experimental validation using synthetic ground truth based on the cell image of Fig. 3(a) is described in the supplementary material S2.

\section{Conclusion}

We proposed in this paper a new principle for contour-to-image registration, the maximal mass confinement (MMC) principle, we derived our MMC algorithm from it and we used it as a basis for a new strategy of hierarchical rigid and locally rigid image registration. This strategy avoids problems associated to previous approaches looking for corresponding confiner pairs, which are sensitive to differences in both images due to noise or different conditions of image acquisition. Our evaluation and comparison with an iconic registration approach show a high accuracy and robustness with respect to such image differences. In particular, the algorithm is able to deal with large local movements. It is simple to implement and further potential of the proposed approach results from its computational speed. Currently, we are working on integrating more sophisticated motion models into our strategy.

\section{References}

1. Pluim, J.P.W., Maintz, J.B.A., Viergever, M.A.: Mutual information based registration of medical images: A survey. IEEE Tr. Med. Im. 22(8), 986-1004 (2003)

2. Kok-Wiles, S.L., Brady, M., Highnam, R.: Comparing mammogram pairs for the detection of lesions. In: 4th Int. Workshop of Dig. Mammography. Kluwer, Dordrecht (1998)

3. Mattes, J., Demongeot, J.: Tree representation and implicit tree matching for a coarse to fine image matching algorithm. In: Taylor, C., Colchester, A. (eds.) MICCAI 1999. LNCS, vol. 1679, pp. 646-655. Springer, Heidelberg (1999)

4. Ding, L., Goshtasby, A., Satter, M.: Volume image registration by template matching. Image and Vision Computing (2001)

5. Hartigan, J.A.: Estimation of a convex contour in two dimensions. J. Amer. Statist. Assoc. 82, 267-270 (1987)

6. Demongeot, J., Cosnard, M., Jacob, C.: Attractors and confiners: Deterministic and stochastic approaches. In: Dynamical Systems - A Renewal of Mechanism. Centennial of Gorge David Birkhoff. World Scientific, Singapore (1986)

7. Mattes, J.: Invariants statistiques et structurels définis par l'arbre de confinement pour le recalage d'images et l'analyse du mouvement. PhD thesis, Grenoble (2000)

8. Studholm, C., Hill, D.L.G., Hawkes, D.J.: An overlap invariant entropy measure of 3D medical image alignment. Pattern Recognition 32, 71-86 (1999) 